\title{
Boosting the capacity of all-organic paper supercapacitors using wood derivatives
}

J esper Edberg, Olle Inganäs, Isak Engquist and Magnus Berggren

The self-archived postprint version of this journal article is available at Linköping University Institutional Repository (DiVA):

http:// urn.kb.se/ resolve?urn=urn:nbn:se:liu:diva-144133

N.B.: When citing this work, cite the original publication.

Edberg, J ., Inganäs, O., Engquist, I., Berggren, M., (2018), Boosting the capacity of all-organic paper supercapacitors using wood derivatives, J ournal of Materials Chemistry A, 6(1), 145-152. https:// doi.org/ 10.1039/ c7ta06810g

Original publication available at:

https:// doi.org/ 10.1039/ c7ta06810g

Copyright: Royal Society of Chemistry http:// www.rsc.org/ 


\title{
Boosting the Capacity of All-Organic Paper Supercapacitors Using Wood Derivatives
}

\author{
Jesper Edberg ${ }^{1,2}$, Olle Inganäs ${ }^{3}$, Isak Engquist ${ }^{1 *}$, Magnus Berggren ${ }^{1,4}$ \\ *Corresponding author: isak.engquist@liu.se \\ ${ }^{1}$ Laboratory of Organic Electronics, ITN, Linköping University, SE-601 74 Norrköping, Sweden \\ ${ }^{2}$ RISE Acreo, SE-601 74 Norrköping, Sweden
}

${ }^{3}$ Biomolecular and Organic Electronics, IFM, Linköping University, SE-581 83 Linköping, Sweden

${ }^{4}$ Stellenbosch Institute for Advanced Studies (STIAS), Wallenberg Research Center at Stellenbosch

University, Stellenbosch 7600, South Africa

\begin{abstract}
Printed and flexible organic electronics is a steadily expanding field of research and applications. One of the most attractive features of this technology is the possibility of large area and high throughput production to form low-cost electronics on different flexible substrates. With an increasing demand for sustainable energy production, low-cost and large volume technologies to store high-quality energy become equally important. These devices should be environmentally friendly with respect to their entire life cycle. Supercapacitors and batteries based on paper hold great promise for such applications due to the low cost and abundance of cellulose and other forest-derived components. We report a thick-film papersupercapacitor system based on cellulose nanofibrils, the mixed ion-electron conducting polymer PEDOT:PSS and sulfonated lignin. We demonstrate that the introduction of sulfonated lignin into the cellulose-conducting polymer system increases the specific capacitance from 110 to $230 \mathrm{Fg}^{-1}$ and the areal capacitance from $160 \mathrm{mFcm}^{-2}$ to $1 \mathrm{Fcm}^{-2}$. By introducing lignosulfonate also into the electrolyte solution, equilibrium, with respect to the concentration of the redox molecule, was established between the electrode and the electrolyte, thus allowing us to perform beyond 700 charge/discharge cycles with no observed decrease in performance.
\end{abstract}




\section{INTRODUCTION}

In recent years, there has been a growing interest in the development of paper-based devices for storage, generation and harvesting of energy. ${ }^{1,2}$ The motivation for using paper is its inherent mechanical strength and flexibility, porosity, large specific surface area, low cost, biocompatibility and its environmental friendliness. Some examples of devices that have been successfully integrated into paper, or that use cellulose as a binder, are supercapacitors, electrochemical batteries, lithium ion batteries, biofuel cells, thermoelectric generators, diodes, photodetectors and nanogenerators. ${ }^{3-8}$ Flexible devices have several advantages over rigid substrates in that they are less sensitive to mechanical stress, can be manufactured in high throughput roll-to-roll production and can be integrated into flexible and wearable electronic devices. The porosity of paper is advantageous in the sense that the included fibers can be coated with electronic charge transporting and redox active materials and that the space between the fibers, as well as the interior of the fibers, can provide ion transport and intercalation. ${ }^{9,10}$ These features allow us to use paper both as a separator and as the electrode material in charge storage devices. ${ }^{11,12}$ Furthermore, the large specific surface area of paper can be used to increase the number of reaction sites and therefore boost the performance of several types of devices. ${ }^{13}$ Besides economical and performance aspects, there is also an increasing demand for green and sustainable technologies, with respect to included materials, manufacturing and recycling. Cellulose is the most abundant biopolymer on earth and by using this material in electronic and electrochemical devices one route towards environmentally friendly digital and energy technologies is constructed. ${ }^{14}$

There are three reported classes of materials that have extensively been explored and also utilized as the electrodes in supercapacitors; carbon, metal oxides and conductive polymers. ${ }^{15-19}$ Carbon is the most commonly used material in commercial supercapacitors due to its abundance and low cost. Although high values of specific capacitance $\left(>400 \mathrm{Fg}^{-1}\right)$ has been reported for carbon nanotube materials in literature ${ }^{20}$, commercial capacitors with activated carbons reach only $25-30 \mathrm{Fg}^{-1} \cdot{ }^{21}$ The ionic conductivity of the carbon electrode is highly dependent on the porosity of the material, and can be limiting. ${ }^{16}$ 
Many metal oxides have been reported in literature to have very high values of specific capacitance, on the order of $1000-2000 \mathrm{Fg}^{-1} \cdot{ }^{22}$ However, the best-performing metal oxides are costly and are therefore generally not used or explored in commercial applications. ${ }^{17}$ Metal oxides also suffer from poor electronic and ionic conductivity as well as short lifetime due to volumetric expansion and contraction during operation that causes cracking. ${ }^{19}$ To achieve good ion intercalation, these materials often require different types of nanostructuring, making upscaling of devices to very large volumes and dimensions difficult.

The third class of materials is the conductive polymers. ${ }^{23-25}$ The advantages of this type of material are its low resistance, mechanical flexibility and processability, which enables large area and volume fabrication at room temperature. Conducting polymers are proven easily composited with paper and provide excellent energy storage or harvesting characteristics in large-scale electrodes. ${ }^{14}$ Some conductive polymers, such as polypyrrole and polyaniline, have shown very high specific capacitance $\left(600^{26}\right.$ and $800^{27} \mathrm{Fg}^{-1}$ respectively), but are unstable and quickly degrade during electrochemical cycling due to volumetric change. ${ }^{16}$ One of the most stable conductive polymers is poly(3,4-ethylenedioxythiophene), or PEDOT. However, the theoretical specific capacitance of this polymer is only $210 \mathrm{Fg}^{-1}$ and typical reported values ranges from $80-90 \mathrm{Fg}^{-1} \cdot{ }^{28,29}$

We have recently demonstrated a composite material of cellulose nanofibrils (CNF) and the conductive polymer system PEDOT:PSS (where PSS is polystyrene sulfonate) that was used to make allorganic paper-supercapacitors. ${ }^{30}$ A study of the microstructure of the material, using AFM, XPS and GIWAXS, revealed that the conductive polymer forms a cladding layer on the cellulose fibrils. We showed that this material system exhibited high electronic and ionic conductivity, good stability and that its performance as a supercapacitor scaled linearly with thickness. These characteristics enabled us to manufacture thick, flexible and self-supporting paper electrodes (Figure 1b) to be used in supercapacitors possessing high charge storage capacity. In this report, we have introduced another redox-active organic material, lignosulfonate (LS), into this material system in order to increase the charge storage capacity of the cathode of the paper-supercapacitors. LS is a derivative of lignin, which is the second most abundant 
biopolymer after cellulose, and is commonly referred to as a waste product of wood pulp during paper making. This cheap and green material can store electrical energy in its phenolic groups by switching between the quinone and hydroquinone states (Figure 1a). Since LS is stable in its hydroquinone state, the molecule can be oxidized to a benzoquinone state by applying a positive potential. This means that the molecule can be used as the positive electrode in a supercapacitor/battery. However, since LS is an electronic insulator, it cannot be used by itself as the electrode material in supercapacitors. Therefore, LS and other phenolic molecules have previously been combined with conductive polymers that enable charge transport. ${ }^{31-33}$ Ajjan et al. demonstrated a PEDOT-LS composite with excellent charge storage capacity and stability.$^{28}$ However, this material system was prepared as thin films on small electrodes using chemical and electrochemical polymerization, techniques which are limited in manufacturing scalability. In this report, we investigate if LS can efficiently be integrated into a scalable and solution processable material systems, which is suitable for large-volume commercial supercapacitor systems. We also address one of the major issues with current energy storage systems based on redox-active organic compounds, namely dissolution of these molecules from the electrodes and into the electrolyte which causes the capacity to degrade over time. We demonstrate a new way of getting around this problem by incorporating the active molecules at high concentration into the electrolyte.

\section{EXPERIMENTAL}

\subsection{Materials}

Poly(3,4-ethylenedioxythiophene):poly(styrenesulfonate) (PEDOT:PSS), Clevios PH 1000, was purchased from Heraeus. Glycerol, dimethyl sulfoxide (DMSO), acetonitrile and perchloric acid $\left(\mathrm{HClO}_{4}\right)$ were purchased from Sigma-Aldrich. Lignosulfonate (LC30, $\left.\mathrm{M}_{\mathrm{W}}=13400\right)$ was purchased from MeadWestvaco. Cellulose nanofibrills (CNF) was produced via high-pressure homogenization of cellulose dispersed in water at 1700 bar. The resulting concentration of the CNF dispersion was $0.41 \mathrm{wt} \%$. The CNF dispersion was provided by Innventia AB. 
Lignosulfonate (LS) was dissolved in $0.1 \mathrm{M} \mathrm{HClO} 4$ at a concentration of $10 \mathrm{mg} / \mathrm{mL}$. The solution was mixed with poly(3,4-ethylenedioxythiophene):poly(styrenesulfonate) (PEDOT:PSS) resulting in solid content weight ratios of 1:0 (PEDOT:PSS solution without lignosulfonate), 2:1, 1:1, 1:2, 1:5 and 1:10 of PEDOT:LS (P:L). The P:L ratios does not include the mass of PSS, and only the mass of PEDOT (PSS excluded) was taken into account for the calculation of specific capacitances.

\subsection{Paper manufacturing}

The conductive paper was prepared by mixing $4.7 \mathrm{~g}$ of PEDOT:PSS-LS mixture with $5.5 \mathrm{~g}$ of CNF solution, 258 mg DMSO and 36 mg glycerol. The solutions were homogenized using a T 10 basic ULTRATURRAX (Laboratory Mixers) after which they were degassed for 1 hour. Finally, the solutions were cast into plastic petri dishes $\left(5.5 \mathrm{~cm}\right.$ diameter) and left to dry at $60^{\circ} \mathrm{C}$ in oven. The two samples with the highest amount of lignosulfonate (1:5 and 1:10) became very brittle and could not be lifted from the petri dish after drying and were therefore not used in any measurements.

\subsection{Sample preparation}

The steps of the sample preparation are shown in Figure 1c. (i) $4 \mathrm{~mm}$ (5 mm in the cycling experiment) diameter disks were cut out of the papers, (ii) carbon fibers were added for the electrical contacts, (iii) carbon paste was added to work as a glue between the carbon fibers and the paper, (iv) another paper disk was added on top of the carbon paste, $(v)$ copper tape was added to the ends of the carbon fibers to ensure a good electrical contact and Kapton tape was added to prevent the electrolyte from reaching the copper. These electrodes were used as the working electrodes (WE) together with a platinum counter electrode (CE) and an $\mathrm{Ag} / \mathrm{AgCl}$ reference electrode (RE) in the three-electrode electrochemical measurements (Figure 1d). The papers were $\sim 50 \mu \mathrm{m}$ thick making the final electrodes $\sim 100 \mu \mathrm{m}$ thick, since two pieces were "glued" together, with an area $12.6 \mathrm{~mm}^{2}$.

\subsection{Measurements}


The sheet resistance of the conductive paper samples was measured using a Keithley 2400 source meter in four-wire sense mode. Strips of the samples were glued on to gold electrodes using carbon paste to ensure a stable electrical contact, and the measurement apparatus was connected to the gold contacts (see Figure SI1 in Supplementary Information). The thickness of the samples was measured using a micrometer screw gauge and the surface roughness was characterized using a surface profilometer (see Supplementary Information). The electronic conductivity $(\sigma)$ of the samples was then calculated using Equation 1, where $R_{S}$ is the sheet resistance and $t$ is the thickness of the samples.

$\sigma=\frac{1}{R_{s} t}$

Electrochemical measurements were performed using an $\mu$ AUTOLAB potentiostat (Metrohm) in a threeelectrode setup with a $\left[0.1 \mathrm{M} \mathrm{HClO}_{4}\right.$ in water]/acetonitrile (9/1) electrolyte. The experimental setup is depicted in Figure 1d. 


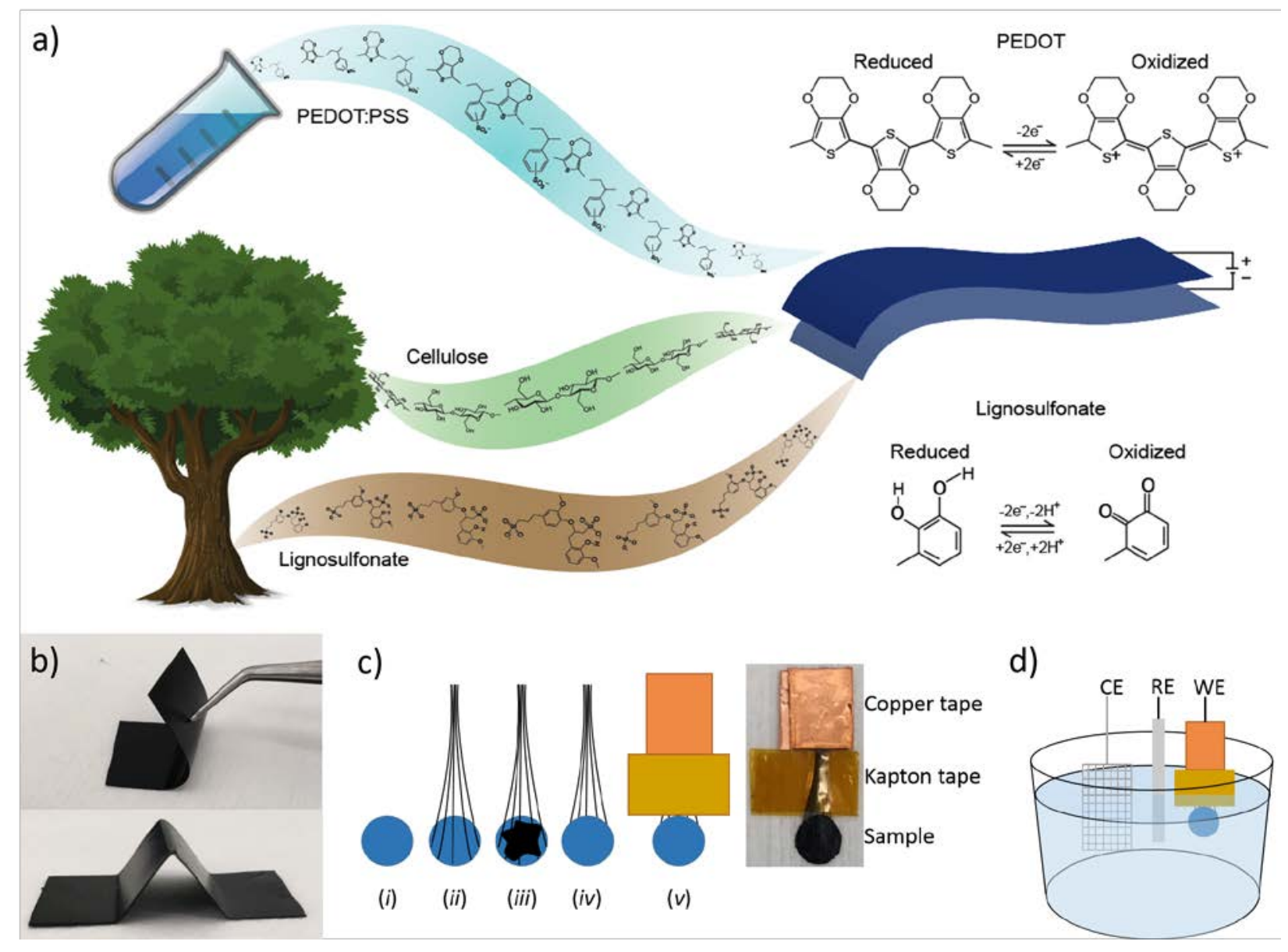

Figure 1. (a) Schematic of the paper supercapacitor with the molecular structures of its constituents, (b) photos of the paper composite being bent and folded, (c) schematic of the sample preparation and a photo of a sample, and (d) a schematic of the three electrode electrochemical setup with working electrode (WE), counter electrode (CE) and reference electrode (RE).

\section{RESULTS \& DISCUSSION}

Figure 2a shows the electronic conductivity of the conductive paper prepared with different loadings of LS. From here on, the samples will be referred to as $\mathrm{P}: \mathrm{L} \_\mathrm{X}: \mathrm{Y}$ (or simply $\mathrm{X}: \mathrm{Y}$ in the graphs), where $\mathrm{X}$ is the PEDOT mass fraction (PSS excluded) and Y is the LS mass fraction. Three samples were prepared for each loading. The electronic conductivity was decreasing with increasing amount of LS, which is simply explained either by the increased thickness of the samples when adding more material and/or by that LS is 
forming insulating cladding phases which limits charge transport in between PEDOT domains. However, the difference in the mass (and thus thickness) of the samples cannot be explained by the addition of LS alone. During the drying process, most of the water and some of the high boiling point organic solvents (DMSO and glycerol) evaporates from the samples. By weighting the samples before and after drying, and then subtracting the mass of the solid components (PEDOT:PSS, NFC and LS), the mass of the solvents left in the samples can be estimated. Figure SI2 in Supplementary Information shows the weight percentage of solvents in samples with different loading of LS. The percentage of solvent increases with the amount of LS indicating that LS slows down the evaporation process or promotes binding of the solvent relatively stronger to the paper. The resulting thickness of the paper (Figure 2b) increases as more and more LS is added and the corresponding higher amount of solvent stored in the paper explains the major part of the drop in electronic conductivity. Also, the surface roughness increased with the addition of LS, as was observed in surface profilometer measurements (see Supplementary Information).

In Figure 2c the sheet resistance of the different samples is given. Since the amount of conductive material for a given area is the same for all samples (the same amount of PEDOT:PSS was added to each sample), the sheet resistance gives a measure of the total charge transport and should only increase if LS or solvents are forming insulating phases in such a way that electronic charge transport through the PEDOT phase is suppressed. The sheet resistance is more or less constant up to a mass fraction $\left(\mathrm{m}_{\mathrm{LS}} / \mathrm{m}_{\mathrm{PEDOT}}\right)$ of 1 , but then increases by $\sim 34 \%$ for a mass fraction of 2 (as compared to mass fraction 0 ). The results indicate that the drop in electronic conductivity, upon introducing LS, is primarily caused by an increase of the thickness of the samples, while charge transport is unaffected up to a mass fraction of 1.

The equivalent series resistance (ESR) is the total resistance of the measurement cell, including the resistance from the sample, contacts and electrolyte as well as interfacial resistances between them. One of the major advantages of supercapacitors based on conductive polymers, over inorganic materials, is their low ESR, which in turn reduces power losses thus giving a high-power density under operation. ${ }^{25}$ The ESR 
of our paper supercapacitors was calculated from the potential drop $(U)$ recorded at the beginning of the galvanostatic discharge measurements using Equation 2:

$$
R_{E S R}=\frac{U}{2 I}
$$

The constant current $(I)$ is multiplied by a factor 2 to account for the instantaneous switch from positive to negative current during the measurement. Figure $2 \mathrm{~d}$ shows that the ESR of the paper supercapacitors, on average, stays relatively constant for all loadings of LS. From the measurements of sheet resistance, the resistance across a sample can be approximated as $0.4 \Omega$, since the samples used in the cell were twice as thick as the samples used in the resistance measurements. This mean that the ESR was two orders of magnitude higher than the resistance of the samples. Some of the resistance comes from the electrolyte between the WE and RE, however, the largest contribution to the ESR comes from the carbon fibers used to contact the samples, which had a resistance of $\sim 10 \Omega \mathrm{cm}^{-1}$ (for the specific wire thickness used in the experiments). Also, the carbon paste used between the fibers and the sample have a relatively high resistivity. Forming a good electrical contact was challenging since the samples contain a large amount of solvents. Several methods of contacting the samples were investigated. Direct contacting onto metal electrodes require the cell to be clamped. This can cause damage to the films since they become more fragile when hydrated by the electrolyte. This method is also impractical when performing three electrode measurements. Another method was to directly contact the samples using crocodile clips. This method was also too destructive and would cause corrosion on the crocodile clip which increased the ESR with time. This indicates that there is much room for improvement, which could be achieved by changing the structure of the measurement cells to improve electrical contact. 

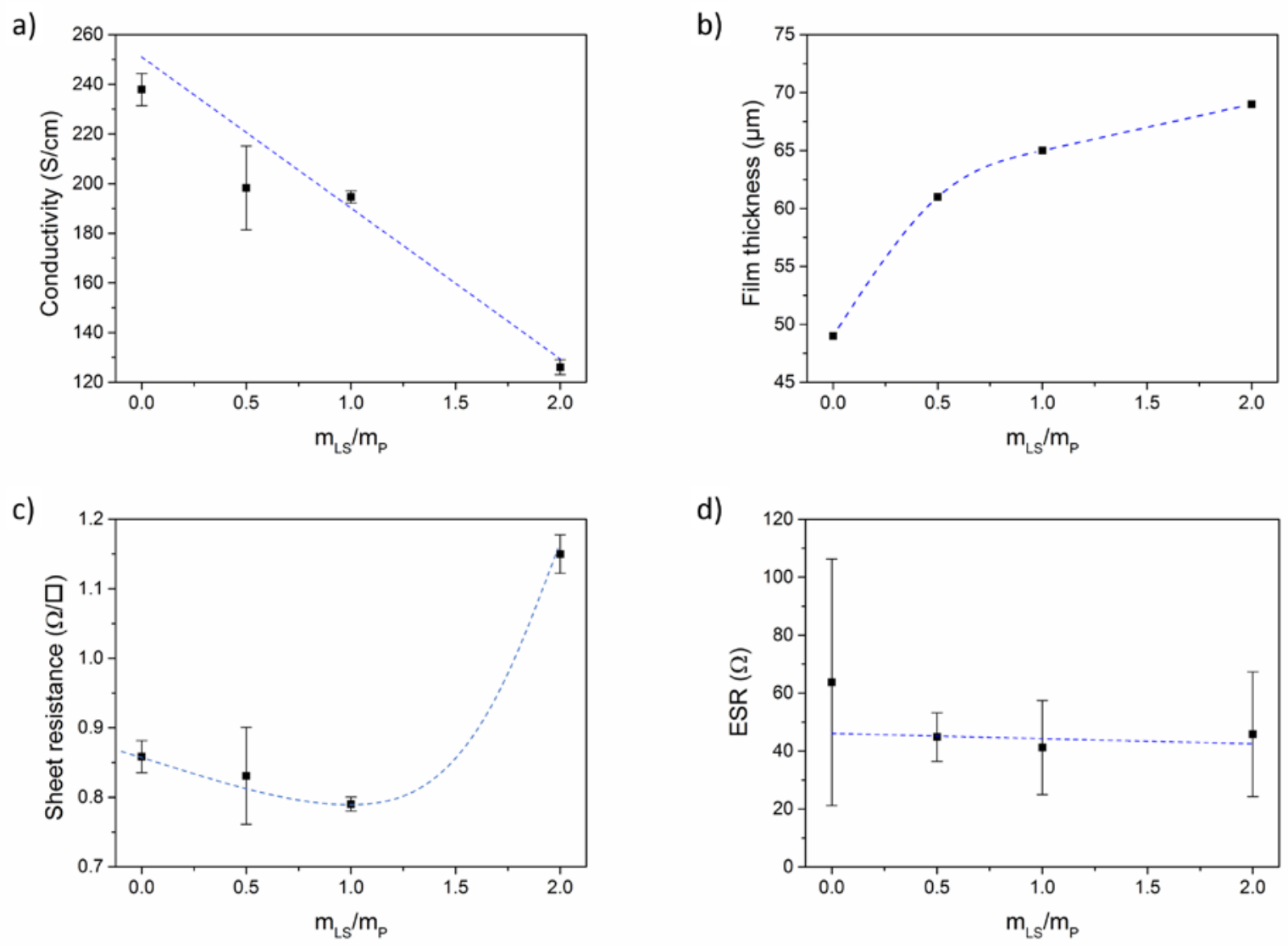

Figure 2. (a) Electronic conductivity, (b) film thickness, (c) sheet resistance and (d) ESR of paper samples with different LS/PEDOT mass ratios. The dashed lines in (a) and (d) are linear fits while the lines in (b) and (c) are included only as a guide to the eye. The statistics in (a), (c) and (d) was performed using five data points for each loading LS loading level.

Cyclic voltammetry was performed at a scan rate of $2 \mathrm{mVs}^{-1}$ (Figure 3a). Electrode materials that contain lignosulfonate displays clear redox peaks, while the sample without lignosulfonate shows pure (pseudo)capacitive charging.

Using chronopotentiometry, the paper electrodes were charged up to $0.7 \mathrm{~V}$ at a current bias of 0.5 $\mathrm{Ag}^{-1}$, calculated on the mass of PEDOT (excluding PSS) and LS, and then discharged to $0.1 \mathrm{~V}$ at the same rate. Figure 3b shows the discharge curves of samples with different LS loading. The samples without LS show a linear discharge curve, indicating an ideal capacitive behavior while samples with LS show a 
nonlinear discharge with a voltage plateau region, indicating LS redox activity. This latter behavior is typical for a battery rather than that of a supercapacitor. However, since the voltage of this hybrid capacitor-battery still have a strong dependence on the state of charge, characterizing the material as a battery is not suitable. Instead, a capacitance is defined within a potential window which contains the electrochemical potential of LS. This method has been previously used for composites of conductive polymers and redox-active molecules. ${ }^{28,31-33}$ However, the choice of the potential window will affect the obtained capacitance, and so, to make the results comparable with previous works, the same potential window as was reported by Ajjan et al. was used in this work. ${ }^{28}$ Complete charge-discharge curves can be found in supplementary information (Figure SI5).

The capacitance $(C)$ was calculated from the galvanostatic discharge curves using the equation $C=\left(I^{*} \Delta t\right) / \Delta V$ where $\mathrm{I}$ is the current, $\Delta t$ is the discharge time and $\Delta V$ is the change in potential. The capacitance was calculated between $V_{\max }$ and $0.1 \mathrm{~V}\left(\Delta V=V_{\max }-0.1 \mathrm{~V}\right)$, where $V_{\max }$ equals $0.7 \mathrm{~V}$ reduced by the IR-drop present at the beginning of the discharge. This IR-drop is found to be on the same level as was found and used by Ajjan et al in their experiments. ${ }^{28}$ Figure 3c and 3d shows the capacitance calculated from five samples of each LS loading. Figure 3c shows the specific capacitance, i.e. the capacitance normalized to the mass of the electroactive materials (PEDOT and LS), and Figure 3d shows the capacitance normalized to the electrode area (areal capacitance). The specific capacitance increased from $110 \mathrm{Fg}^{-1}$ (P:L_1:0) to a maximum of $230 \mathrm{Fg}^{-1}$ (P:L_1:1) and then plateaued while the areal capacitance increased linearly from $160 \mathrm{mFcm}^{-2}$ (P:L_1:0) to $1 \mathrm{Fcm}^{-2}$ (P:L_1:2). The mass of PSS was not considered in the calculation of specific capacitance for two reasons: 1) PSS is a large polymer counter ion. If its mass is taken into the account, it cannot properly be compared to other PEDOT systems with small-sized and mobile counter ions, like chloride or tosylate. 2) In solution, there is an excess of PSS and one role of DMSO is to remove this excess. The amount of PSS that is ionically coupled to PEDOT is therefore much smaller than the total amount of PSS in the sample. Also, during the processing of the samples and in the electrolyte solution during measurements, PEDOT is exposed to small anions that can replace PSS as the counter ion 
for PEDOT, making the final amount of PSS ionically bound to PEDOT difficult to estimate. By this method of presenting the data, Figure 3c and 3d give complementary information about the system; one reducing the charge storage mechanism to its most fundamental components (Figure 3c) and the other showing the change in the total capacitance (Figure 3d). However, to give the full picture, values of the specific capacitance calculated using different mass components can be found in Table SI1 in Supplementary Information.

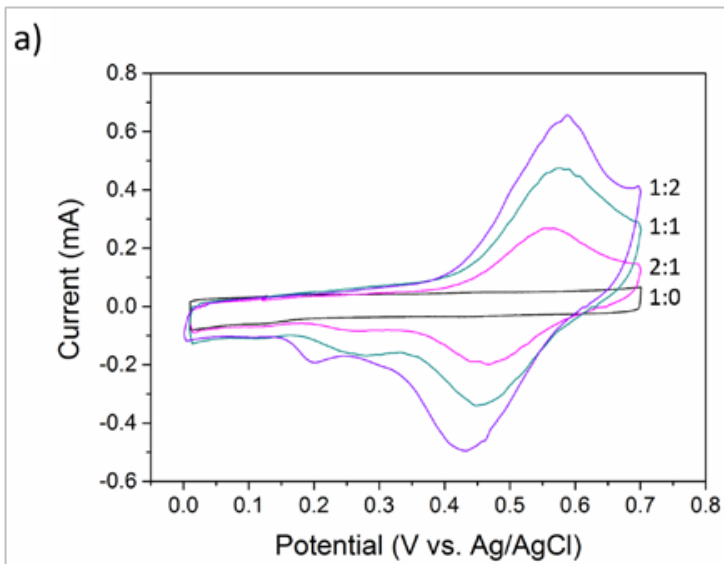

c)

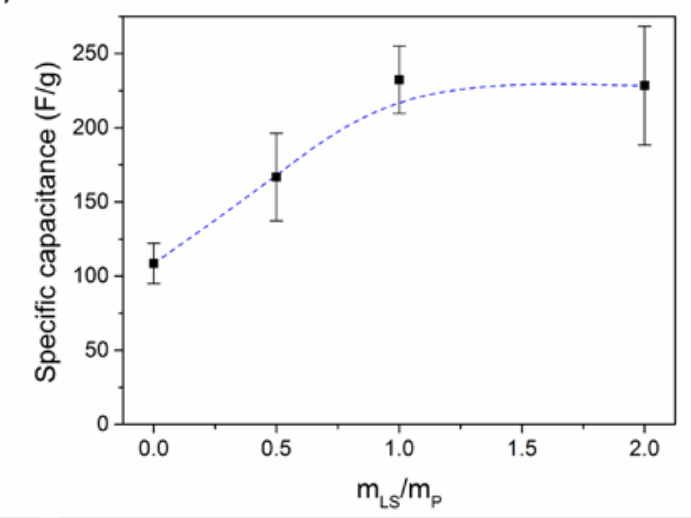

b)

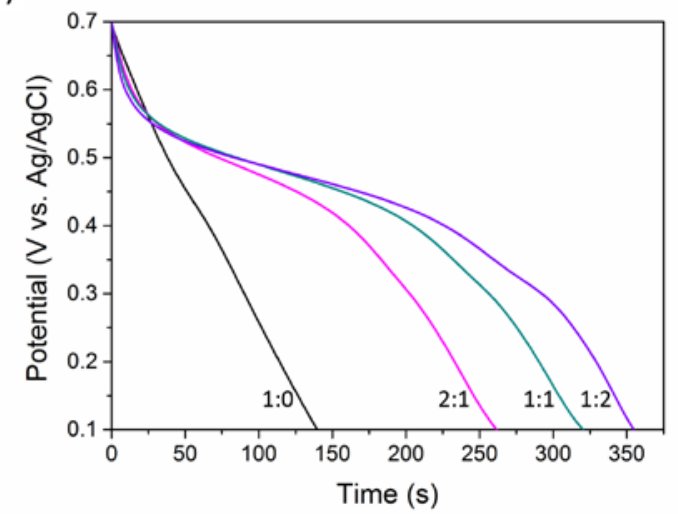

d)

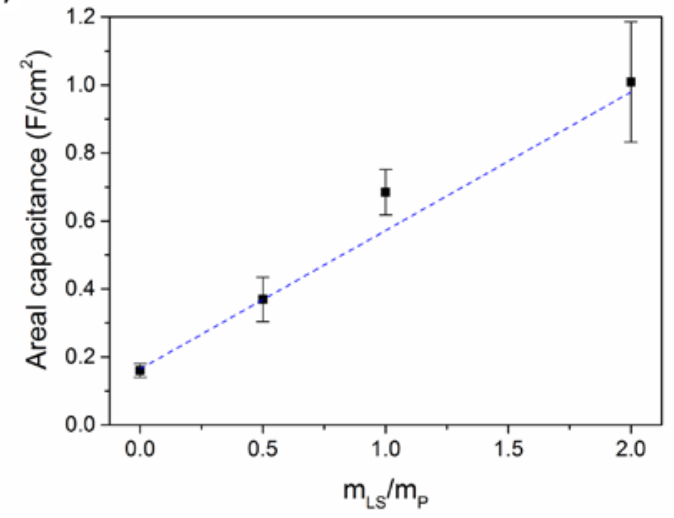

Figure 3. (a) Cyclic voltammetry (scan rate $2 \mathrm{mV} / \mathrm{s}$ ), (b) galvanostatic discharge ( $0.5 \mathrm{Ag}^{-1}$ current density), (c) specific capacitance calculated using the mass of PEDOT and LS, and (d) areal capacitance of samples with different LS/PEDOT mass ratios. The dashed line in (d) is a linear fit while the line in (c) is included only as a guide to the eye. The statistics in (c) and (d) was done using five data points for each LS loading level. 
Galvanostatic discharge measurements were further performed at different current densities (0.5-8 $\left.\mathrm{Ag}^{-1}\right)$ to investigate the rate capability of the composite. Due to the high currents in some measurements (caused by the large mass of the samples), the potential drop became significant and spanned over a large part of the total potential window. To get around this issue, the samples were charged to $0.9 \mathrm{~V}$ instead of $0.7 \mathrm{~V}$ prior to discharge. In doing so, the potential drop was confined to the potential window 0.7-0.9 V. However, for the P:L_1:2 sample at $8 \mathrm{Ag}^{-1}$, the potential drop became too large even with the expanded potential window. This measurement was therefore omitted. Further increasing the potential window beyond $0.9 \mathrm{~V}$ was not possible since signs of overoxidation of PEDOT could be observed for such high potentials. Figure 4a shows the specific capacitance for different current densities. The results show a constant capacitance up to $8 \mathrm{Ag}^{-1}$ for all samples except P:L_1:2, indicating excellent rate capability. The highest charging rate corresponds to 7 seconds charging/discharging times for the P:L_1:0 sample and 15 seconds for the P:L_1:1 sample. The areal capacitance for different current densities can be found in Supplementary Information (Figure SI3).

The stability of the paper composite was investigated by cycling a sample (P:L_2:1) 700 times at 1 $\mathrm{Ag}^{-1}$ using chronopotentiometry. Figure $4 \mathrm{c}$ shows $\mathrm{C} / \mathrm{C}_{0}$ (expressed in \%) where $\mathrm{C}$ is the capacitance of each cycle and $\mathrm{C}_{0}$ is the initial capacitance. The capacitance drops to $45 \%$ over 700 cycles, where the larger part of the drop occurs during the first 100 cycles. This level corresponds to a sample with very little or even no LS. The drop in capacitance during cycling is attributed to dissolution of LS into the electrolyte. This theory was corroborated by a discoloration of the electrolyte solution during the measurement, as can be seen in the inset of Figure 4b. To determine if the discoloration was in fact caused by leakage of LS, we measured the UV-Vis spectra of the electrolyte before and after a sample was cycled 500 times. A measurement of LS dissolved in electrolyte was also taken as a reference. From Figure $4 \mathrm{~b}$ it is clear that the electrolyte after cycling looks qualitatively similar to the LS reference sample. The deviation in the two spectra is believed to be caused by the conversion of phenolic hydroxyl and methoxyl groups into quinone groups during the 
oxidation process occurring during cycling. ${ }^{34}$ This results in a darker color compared to pristine LS, resulting in a higher absorbance.

The leakage is an effect of the high ionic conductivity of the nanopaper which is important for achieving high power density, but also easily lets ions leave the material. To stop the capacitance from degrading by LS diffusion, the initial giant gradient of LS between the sample and electrolyte must be equilibrated or, even to some extent, reversed. To this end, another sample with the same LS loading was cycled 700 times but now in an electrolyte containing $50 \mathrm{mg} / \mathrm{mL}$ of LS. The results are given in Figure 4c and shows that the addition of LS to the electrolyte not only suppress the degradation completely, but in fact increases the capacitance by 50\%. This is caused by the concentration of LS in the electrolyte initially being higher than inside the electrode sample, thus producing a net influx of LS into the electrodes, increasing the resulting capacitance.

The strategy of adding LS into the electrolyte was further explored for samples with an even higher LS loading (P:L_1:2). Figure 4d shows that in an electrolyte with no LS, the capacitance drops to 75\% over 20 cycles. With an electrolyte including $50 \mathrm{mg} / \mathrm{mL}$ of LS, the capacitance still dropped, but only to $90 \%$ of the initial value. At this point, the concentration of LS in the electrolyte was still smaller (5\%) as compared to the concentration within the sample electrode ( 9\%). At $100 \mathrm{mg} / \mathrm{mL}$ (or 10\%), the concentration in the electrolyte was higher than that within the sample electrode and the capacitance was found to slightly increase instead. 
a)

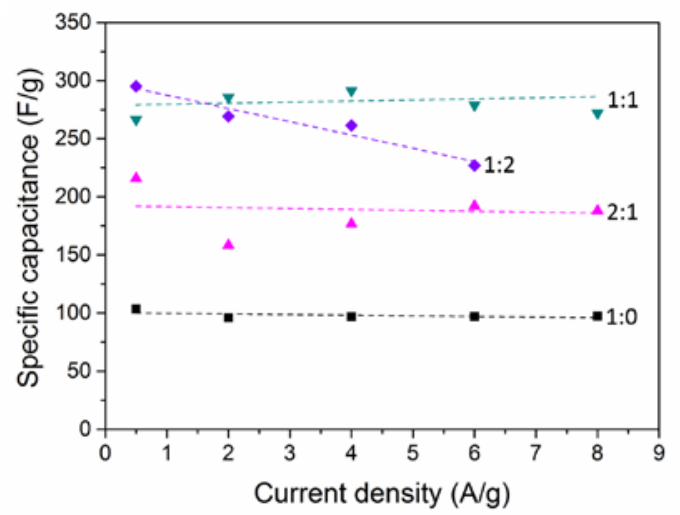

c)

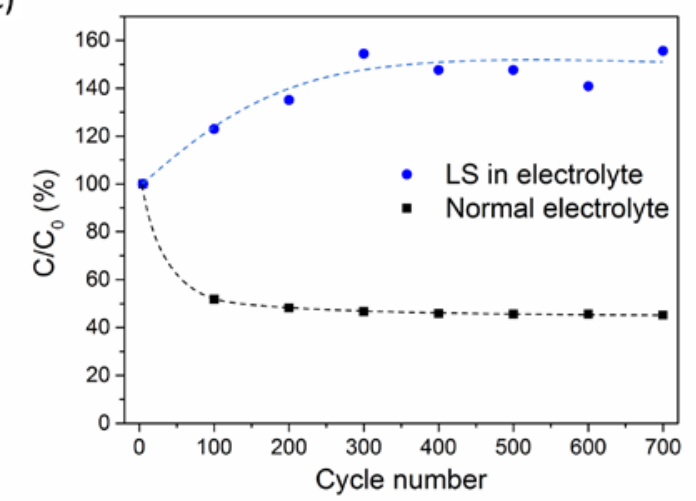

b)

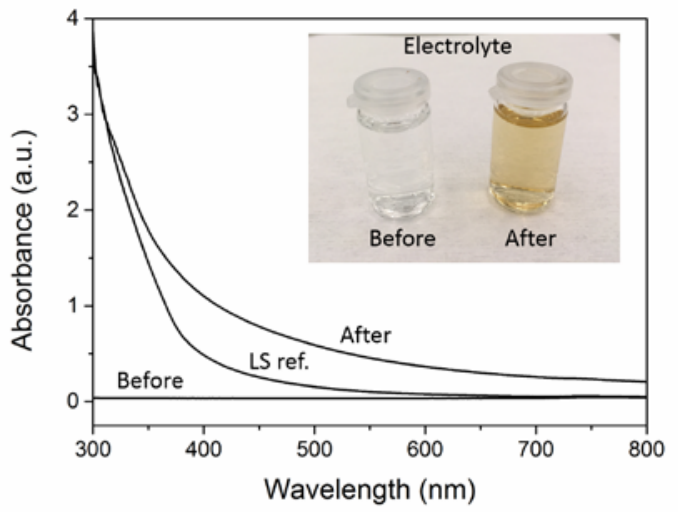

d)

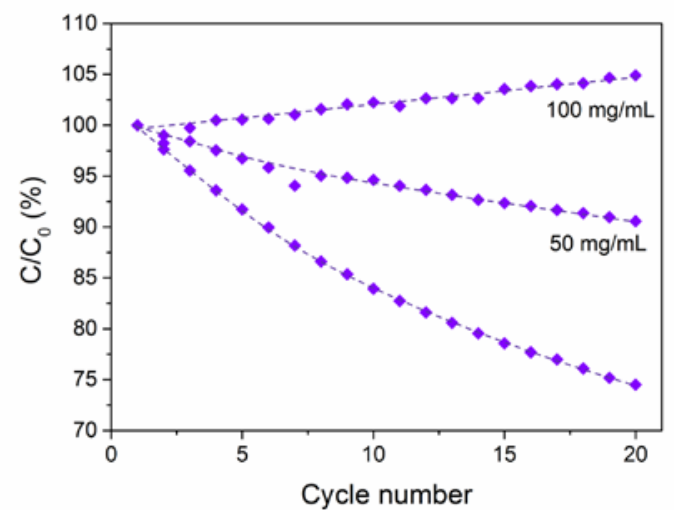

Figure 4. (a) Specific capacitance vs. current density for samples with different LS loading. (b) UV-Vis spectra of electrolyte solution before and after cycling, as well as a reference sample with LS dissolved in the electrolyte. The inset show a photo of the electrolyte solution before and after cycling measurements. (c) capacitance cycling over 700 cycles and (d) capacitance cycling in normal electrolyte and electrolyte with 50 and $100 \mathrm{mg} / \mathrm{mL}$ LS. The dashed lines in (a) are linear fits while the lines in (c) and (d) are included only as guides to the eye. The specific capacitance in (a) was calculated using the mass of PEDOT and LS.

\section{CONCLUSIONS}

The introduction of lignosulfonate (LS) into CNF-PEDOT paper supercapacitors resulted in an increase in the areal capacitance and specific capacitance on the positive electrode. The results show that LS is compatible with polymer- and paper-based supercapacitor systems possible to scale into large production and prototype volumes and therefore has the potential to be used in commercial supercapacitor applications for giant-scale electrical power applications. The charge-discharge characteristics of the 
electrode material start out as that of an ideal capacitor when no LS is added but becomes more and more similar to those of a battery at increasing loading of LS. The ESR of the devices was not affected by the introduction of LS and the corresponding sheet resistance measurements of the paper electrode samples indicate that LS has very little effect on the charge transport of the system. The material exhibited excellent rate performance which allowed us to charge the electrodes at high current densities of $8 \mathrm{Ag}^{-1}$ with little or no loss in capacitance. However, a loss in capacitance in the samples containing LS was seen when cycling the devices and was attributed to LS leaking out from the electrode samples into the electrolyte. This leakage was completely suppressed by adding LS also into the electrolyte solution at a concentration matching that of LS inside the electrode. This strategy solves one of the major problems with energy storage systems based on organic molecules, where dissolution of the molecules into the electrolyte limits the lifetime of the device. Other future strategies to prevent such leakage may include to crosslink LS or protecting the electrodes with membranes that are only permeable to small ions.

\section{ACKNOWLEDGEMENTS}

This work was financially supported by The Knut and Alice Wallenberg foundation (Power Paper project) KAW 2011.0050, the Swedish Foundation for Strategic Research (GMT14-0058), the Önnesjö Foundation, the Swedish Energy Agency, the Advanced Functional Materials Center at Linköping University, and RISE Research Institutes of Sweden.

\section{CONFLICTS OF INTEREST}

The authors declare that they have no competing interests. 


\section{REFERENCES}

1. M. M. Pérez-Madrigal, M. G. Edo and C. Alemán, Green Chemistry, 2016, 18, 5930-5956.

2. Y. Z. Zhang, Y. Wang, T. Cheng, W. Y. Lai, H. Pang and W. Huang, Chemical Society Reviews, 2015, 44, 5181-5199.

3. Y. Lin, D. Gritsenko, Q. Liu, X. Lu and J. Xu, ACS Applied Materials and Interfaces, 2016, 8, 2050120515.

4. T. H. Nguyen, A. Fraiwan and S. Choi, Biosensors and Bioelectronics, 2014, 54, 640-649.

5. D. Sun, X. Yan, J. Lang and Q. Xue, Journal of Power Sources, 2013, 222, 52-58.

6. B. Andres, S. Forsberg, A. P. Vilches, R. Zhang, H. Andersson, M. Hummelgård, J. Bäckström and H. Olin, Nordic Pulp and Paper Research Journal, 2012, 27, 481-485.

7. S. Mats, T. Daniel, G. Hjalmar, S. Anurak, D. Dina, B. Magnus and P. J. Magnus, Flexible and Printed Electronics, 2016, 1, 044003.

8. N. Sani, X. Wang, H. Granberg, P. Andersson Ersman, X. Crispin, P. Dyreklev, I. Engquist, G. Gustafsson and M. Berggren, Scientific Reports, 2016, 6, 28921.

9. Z. Gui, H. Zhu, E. Gillette, X. Han, G. W. Rubloff, L. Hu and S. B. Lee, ACS Nano, 2013, 7, 6037-6046.

10. X. Chen, H. Zhu, C. Liu, Y. C. Chen, N. Weadock, G. Rubloff and L. Hu, J. Mater. Chem. A, 2013, 1, 8201-8208.

11. L. Hu and Y. Cui, Energy \& Environmental Science, 2012, 5, 6423-6435.

12. L. Hu, H. Wu, F. La Mantia, Y. Yang and Y. Cui, ACS Nano, 2010, 4, 5843-5848.

13. L. Zhang, P. Zhu, F. Zhou, W. Zeng, H. Su, G. Li, J. Gao, R. Sun and C.-p. Wong, ACS Nano, 2016, 10, 1273-1282.

14. D. Tobjörk and R. Österbacka, Advanced Materials, 2011, 23, 1935-1961.

15. Z. Wu, Y. Zhu and X. Ji, J. Mater. Chem. A, 2014, 2, 14759-14772.

16. G. Wang, L. Zhang and J. Zhang, Chemical Society Reviews, 2012, 41, 797-828.

17. R. Kötz and M. Carlen, Electrochimica Acta, 2000, 45, 2483-2498.

18. M.-T. Lee, J.-K. Chang, Y.-T. Hsieh and W.-T. Tsai, Journal of Power Sources, 2008, 185, 1550-1556.

19. J. Jiang, Y. Li, J. Liu, X. Huang, C. Yuan and X. W. Lou, Advanced Materials, 2012, 24, 5166-5180.

20. M. Hamedi, E. Karabulut, A. Marais, A. Herland, G. Nyström and L. Wågberg, Angewandte Chemie International Edition, 2013, 52, 12038-12042.

21. V. V. N. Obreja, A. Dinescu and A. C. Obreja, International Review of Electrical Engineering, 2010, 5, 272-281.

22. Z. Yu, L. Tetard, L. Zhai and J. Thomas, Energy \& Environmental Science, 2015, 8, 702-730.

23. I. Shown, A. Ganguly, L.-C. Chen and K.-H. Chen, Energy Science \& Engineering, 2015, 3, 2-26.

24. M. Mastragostino, C. Arbizzani and F. Soavi, Journal of Power Sources, 2001, 97-98, 812-815.

25. G. A. Snook, P. Kao and A. S. Best, Journal of Power Sources, 2011, 196, 1-12.

26. D. P. Dubal, S. H. Lee, J. G. Kim, W. B. Kim and C. D. Lokhande, Journal of Materials Chemistry, 2012, 22, 3044-3052.

27. T. C. Girija and M. V. Sangaranarayanan, Synthetic Metals, 2006, 156, 244-250.

28. F. N. Ajjan, N. Casado, T. Rębiś, A. Elfwing, N. Solin, D. Mecerreyes and O. Inganäs, J. Mater. Chem. A, 2016, 4, 1838-1847.

29. C. Karlsson, J. Nicholas, D. Evans, M. Forsyth, M. Strømme, M. Sjödin, P. C. Howlett and C. PozoGonzalo, ChemSusChem, 2016, 9, 2112-2121.

30. A. Malti, J. Edberg, H. Granberg, Z. U. Khan, J. W. Andreasen, X. Liu, D. Zhao, H. Zhang, Y. Yao, J. W. Brill, I. Engquist, M. Fahlman, L. Wågberg, X. Crispin and M. Berggren, Advanced Science, 2016, 3, n/a-n/a.

31. S. Admassie, F. N. Ajjan, A. Elfwing and O. Inganas, Materials Horizons, 2016, 3, 174-185. 
32. G. Milczarek and O. Inganäs, Science, 2012, 335, 1468-1471.

33. M. Wagner, T. Rębiś and O. Inganäs, Journal of Power Sources, 2016, 302, 324-330.

34. J. Wang, Y. Deng, Y. Qian, X. Qiu, Y. Ren and D. Yang, Green Chemistry, 2016, 18, 695-699. 


\section{Boosting the Capacity of All-Organic Paper Supercapacitors Using Wood Derivatives \\ Supplementary Information}

\section{Four-wire resistance:}

The conductivity of the paper samples was measured using the four-wire resistance method. Four gold contacts were evaporated onto glass slides. Strips of the paper samples were then glued to the metal contacts using a carbon ink to ensure good electrical contact. Figure SI1 shows a picture of one of the samples used for resistance measurements.

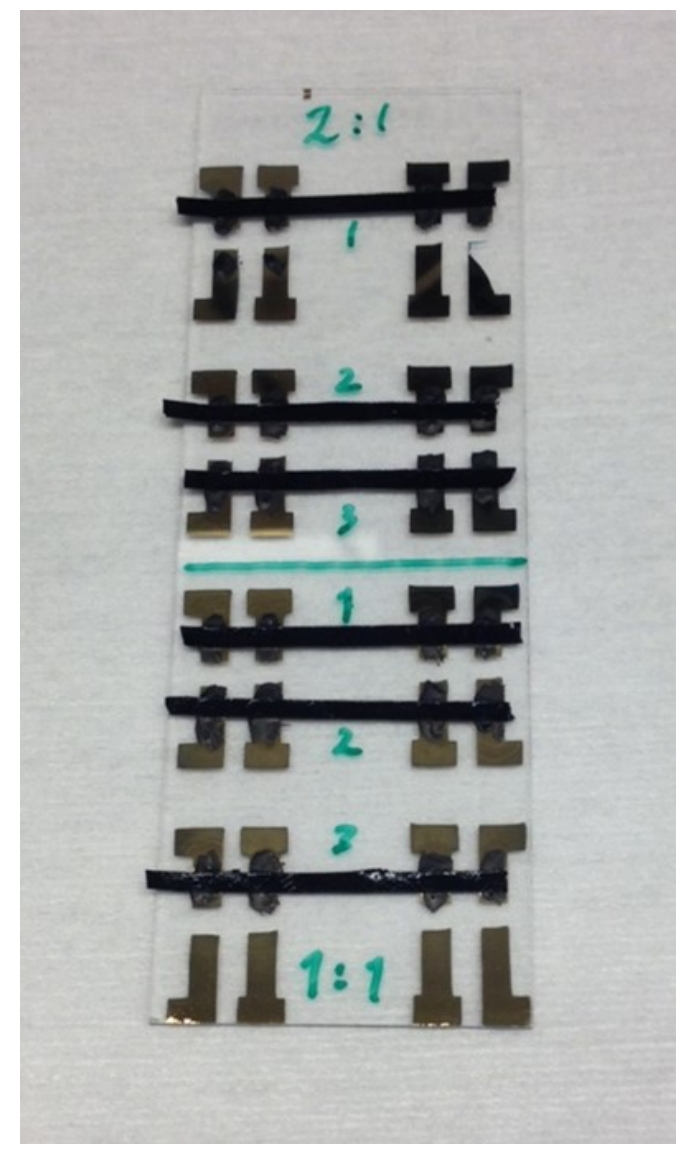

Figure SI1: Picture showing one of the samples used for conductivity measurements. 


\section{Solvent content:}

The thickness of the nanopaper films increases with the amount of LS in the samples (Figure 2b). This can be explained by the combination of adding more LS and the accompanied increase in the amount of solvents (DMSO, glycerol and water) which stay in the samples. The solvent content of the samples was estimated by weighing the samples after drying and comparing the mass to the known amount of solid material (PEDOT:PSS, CNF and LS).

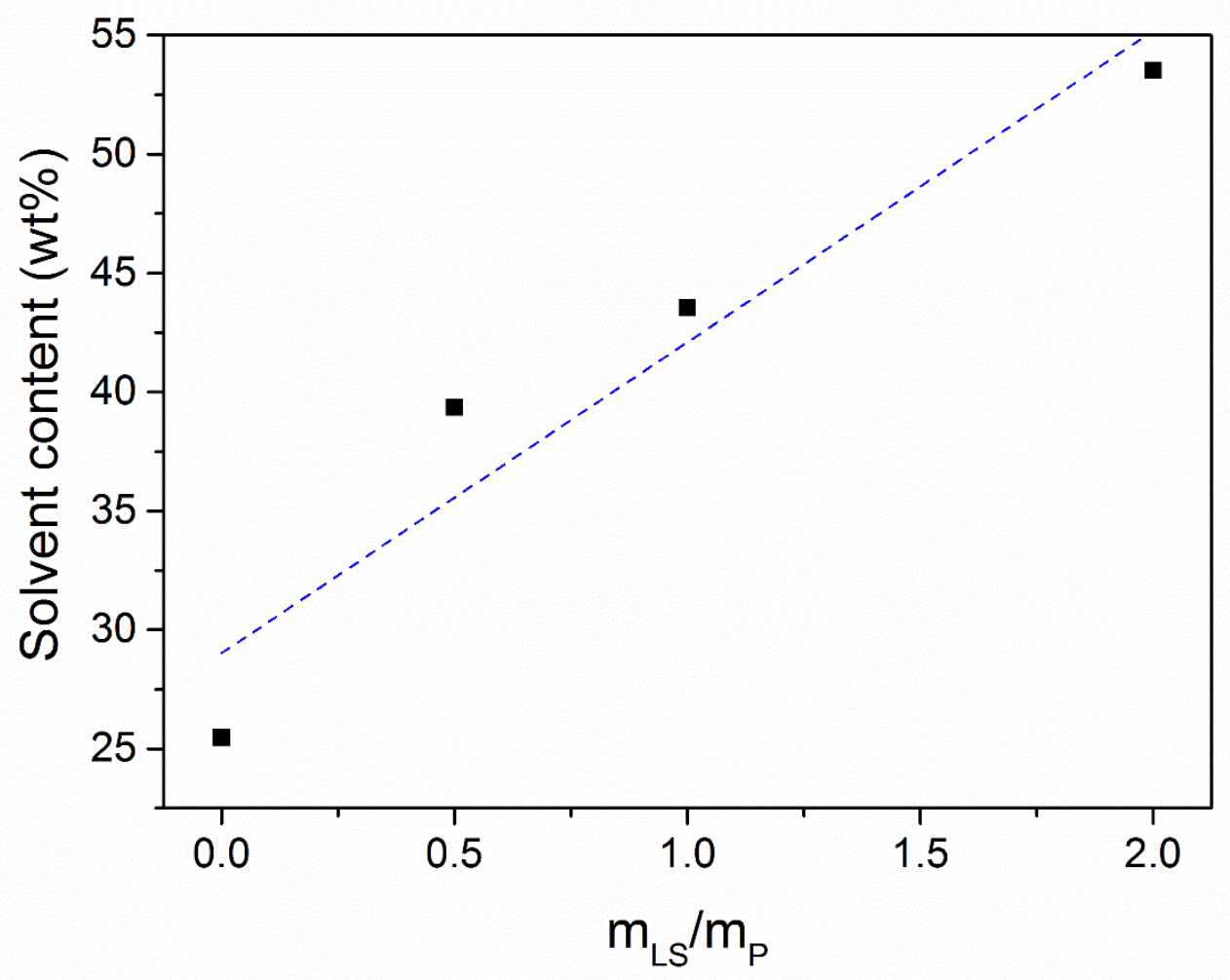

Figure SI2: Solvent content (DMSO, glycerol and water) of paper samples with different amount of LS after drying. 


\section{Capacitance vs. current density:}

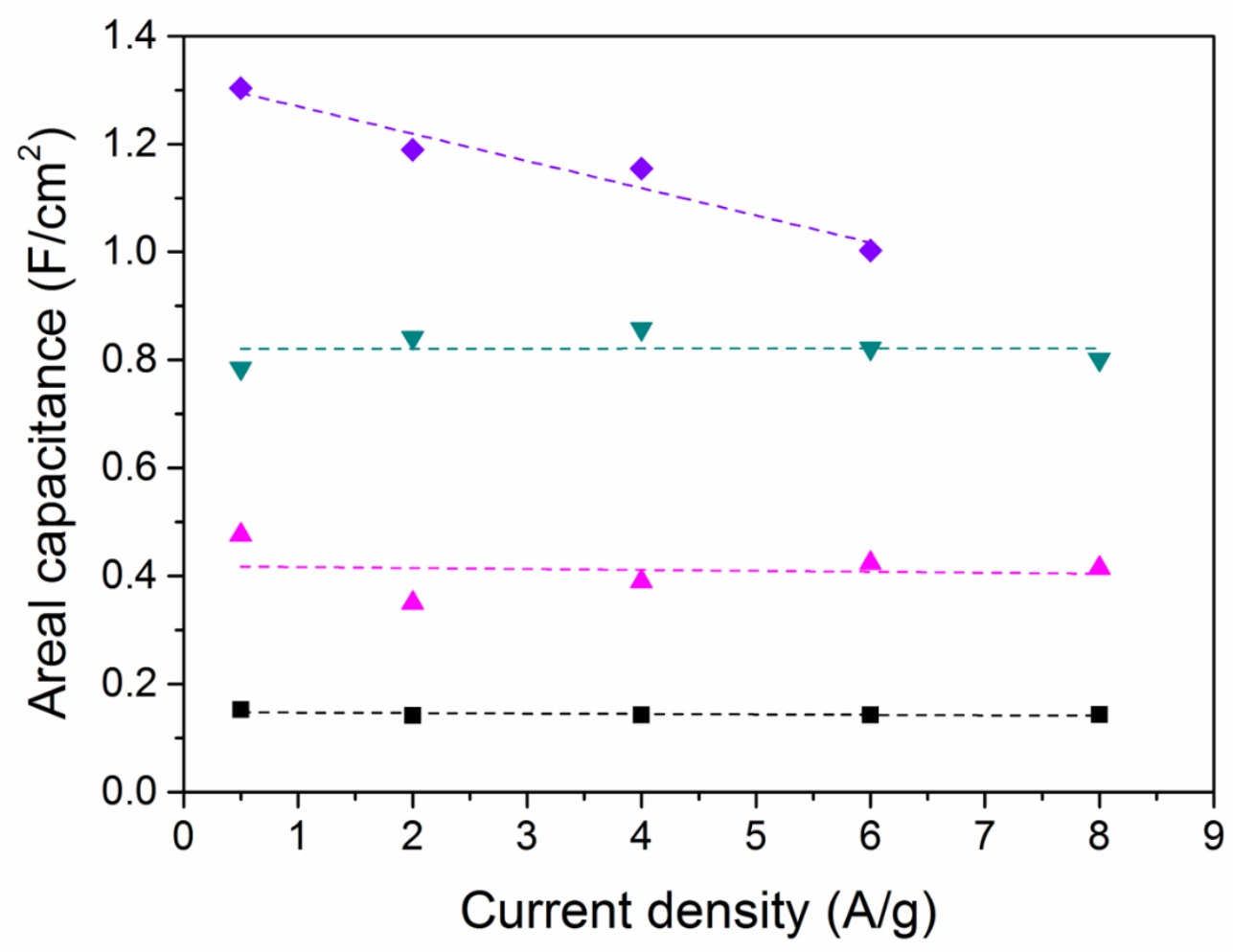

Figure SI3. Areal capacitance for samples of different loading of LS measured at different current densities.

\section{Specific capacitance:}

In a three-electrode electrochemical measurement, the (gravimetric) specific capacitance is the capacitance of an electrode normalized by its weight. When the electrode consists of a composite material with both electronically active and inactive materials, there are different ways of performing the normalization which will show different aspects of the system. On the other hand, when a full device (two-electrode measurement) is characterized, the full mass of the device (including metal collectors, separators, electrolyte, etc.) is usually included in the calculations. The specific capacitance calculated in a three-electrode measurement is $\sim 4$ times higher than the value obtained from a twoelectrode measurement (assuming that both electrodes have equal mass and capacitance). This comes from the fact that only half of the voltage and half the mass is used in the calculations in a threeelectrode measurement compared to a two-electrode measurement.

In the CNF-PEDOT:PSS-LS composite, the electronically active materials are PEDOT and LS. The material also contains the solvents DMSO and glycerol, but these materials are assumed to leak out into the electrolyte during the measurements. Table SI1 present the specific capacitance of Figure 2c calculated in different ways: normalized to the mass of PEDOT+LS, PEDOT+PSS+LS or PEDOT+PSS+LS+CNF, respectively. The mass ratios of PEDOT:PSS, CNF, glycerol and DMSO (excluding LS), is as follows: 13.2/6.1/9.5/68.2 (wt\%) respectively. The mass ratio of PEDOT and PSS is 1:2.5. The mass of PEDOT:PSS in a sample (4 mm diameter) is $650 \mu \mathrm{g}$. 


\begin{tabular}{|c|c|c|c|}
\hline $\begin{array}{c}\text { Mass ratio } \\
\left(m_{L S} / m_{\text {PEDOT })}\right.\end{array}$ & $\begin{array}{c}\text { Specific capacitance } \\
(\mathrm{F} / \mathrm{g}) \\
(\mathrm{PEDOT}+L S)\end{array}$ & $\begin{array}{c}\text { Specific capacitance } \\
(\mathrm{F} / \mathrm{g})\end{array}$ & $\begin{array}{c}\text { Specific capacitance }(\mathrm{F} / \mathrm{g}) \\
(\mathrm{PEDOT}+P S S+L S+C N F)\end{array}$ \\
\hline 0 & 109 & 31 & 21 \\
\hline 0.5 & 167 & 63 & 45 \\
\hline 1 & 233 & 103 & 76 \\
\hline 2 & 228 & 124 & 96 \\
\hline
\end{tabular}

Table SI1. Specific capacitance calculated using different components of the total electrode mass.

\section{Surface roughness:}

The surface roughness of samples with (P:L_1:2) and without (P:L_1:0) LS was measured using a Dektak3ST surface profilometer (Veeco). Both the top and bottom side of the samples (with respect to how they were dried) were characterized. Figure SI4 shows the profile of two samples over a distance of $1000 \mu \mathrm{m}$. The sample without LS had a smaller surface roughness ( $\sim 3 \mu \mathrm{m}$ for the top and $\sim 0.8 \mu \mathrm{m}$ for the bottom) compared to the sample with LS ( 8 $\mu \mathrm{m}$ for both the top and bottom).
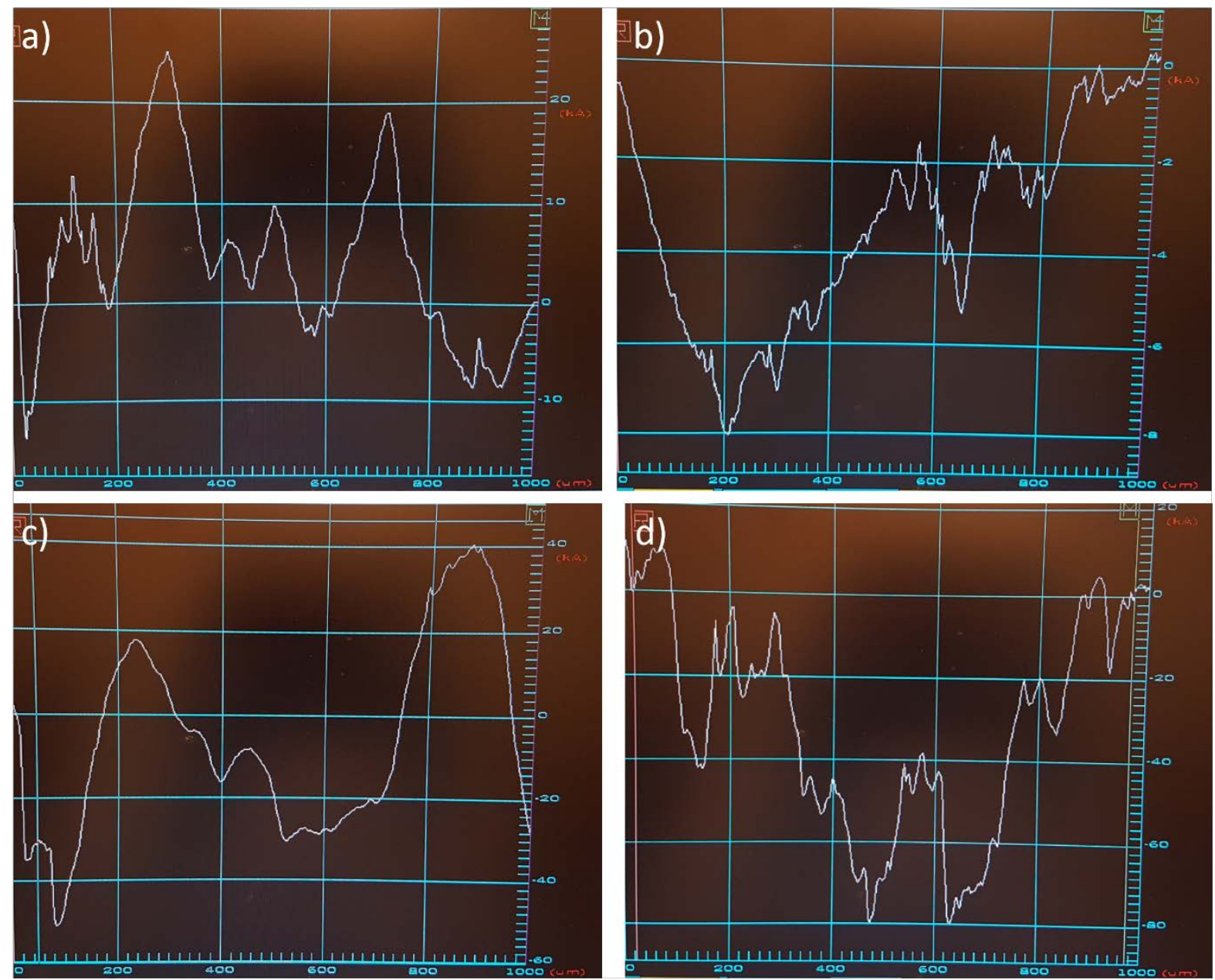

Figure SI4. Surface profile of the top and bottom of samples with or without lignosulfonate. (a) P:L_1:0 top surface, (b) P:L_1:0 bottom surface, (c) P:L_1:2 top surface, and (d) P:L_1:2 bottom surface. 


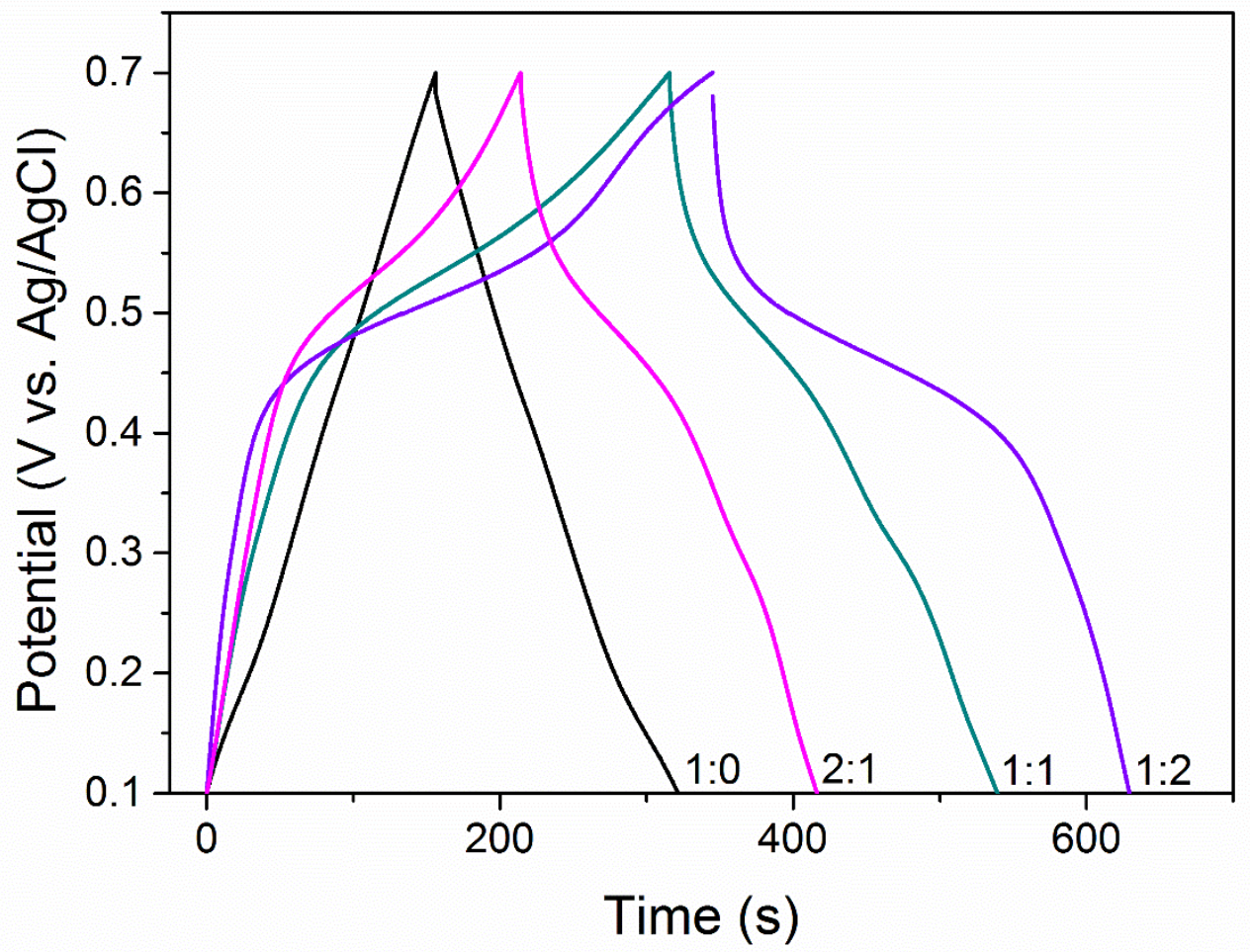

Figure SI5. Galvanostatic charge-discharge curves of samples with different mass ratios of PEDOT and LS (PEDOT:LS). 